TITLE:

\title{
Ising Models, Julia Sets and Similarity of the Maximal Entropy Measures
}

$\operatorname{AUTHOR}(\mathrm{S})$ :

Ishii, Yutaka

\section{CITATION:}

Ishii, Yutaka. Ising Models, Julia Sets and Similarity of the Maximal Entropy Measures. 数理 解析研究所講究録 1994, 863: 1-12

ISSUE DATE:

1994-03

URL:

http://hdl.handle.net/2433/83886

RIGHT: 


\title{
Ising Models, Julia Sets and Similarity of the Maximal Entropy Measures
}

\author{
Yutaka Ishii (石井 豊) \\ Department of Mathematics \\ Faculty of Science \\ Kyoto University
}

\section{Introduction}

In this talk, we study a close relationship between the phase transition of the Ising model and fractal structure of Julia set associated with the Ising model.

Phase transition is formulated as non-analyticity of some physical quantities like free energy $\mathcal{F}$ as a function of some thermodynamical parameters such as temperature. So it is important to know where $\mathcal{F}$ is analytic and how $\mathcal{F}$ behaves near non-analytic (critical) points, because it determines the type of the phase transition. To characerize the phase transition we inrtroduce the following quantity. The critical exponent in the low temperature region of order $l \in \mathbf{N}$ is defined by

$$
\alpha^{(l)} \equiv \lim _{t \nearrow t_{c}} \frac{\log \left|\mathcal{F}^{(l)}(t)\right|}{-\log \left|t-t_{c}\right|}
$$

where $t_{c}$ is the critical temperature and $\mathcal{F}^{(l)}$ is the $l$-th derivative of $\mathcal{F}$.

On the other hand, we can consider a complex dynamical system associated with our model (called the renormalization group transformation). This transformation is a map on parameter (temperature) space corresponding to the "coarse grainning" of the model $[2,3]$. Julia set of the renormalization group reveals a certain similarity near $t_{c}$. 
The purpose of this paper is to show a relationship between two theories, statistical mechanics and complex dynamical systems. Historically, [8] made such a relationship for the first time. In [8], it was shown that all the singularities of $\mathcal{F}$ lie on the Julia set of the renormalization group transformation. Our main results are more quantitative relationship between the critical exponent and the "fractal dimension" at $t_{c}$.

Theorem A The free energy can be represented as the logarithmic potential of the maximal entropy measure $\mu$ of the renormalization group.

$$
F(t)=b \int_{J(f)} \log (t-z) d \mu(z)+C
$$

Using this representation, we can show the following.

Theorem B Let $l$ be so large that $\left(f^{\prime}\left(t_{c}\right)\right)^{l}>2 b$, then we have

$$
\alpha^{(l)}=l-\frac{\log 2 b}{\log f^{\prime}\left(t_{c}\right)}
$$

Moreover $\frac{\log 2 b}{\log f^{\prime}\left(t_{c}\right)}$ represents a similarity of $J(f)$ at $t_{c}$.

Thus, Theorem B says that the phase transition reflects the fractal structure of $J(f)$.

\section{$2 \quad$ Ising Model on Diamond-like Hierarchical Lattices}

For the study of phase transition, various models are considered. Especially, exactly solvable models are deeply investigated. For example, two dimensional Ising model with Onsager's solution and Baxter's six or eight vertex models and so on.

But here we study another exactly solvable model, Ising model on diamondlike hierarchical lattices. The diamond-like hierarchical lattices is the sequence of graphs $\left\{\Gamma_{n}\right\}$ defined as follows. For a fixed integer $b$ greater than one, we shall define two lattices $\Gamma_{G}=\left\{B_{G}, V_{G}\right\}$ (generator) and $\Gamma_{0}=$ 
$\left\{B_{0}, V_{0}\right\}$ (initial lattice) where $B_{*}$ denotes the set of all bonds of $\Gamma_{*}$, and $V_{*}$ denotes the set of all vertices of $\Gamma_{*}$. $\Gamma_{0}$ consists of two vertices and one bond connecting them. To obtain $\Gamma_{G}$, we insert $b$ inner vertices between the two outer ones such that each bond connects an inner vertex and an outer one (see Figure ). When $\Gamma_{n}$ is constructed, $\Gamma_{n+1}$ is obtained by replacing each element of $B_{n}$ by $\Gamma_{G}$. We call $\Gamma_{n}=\left\{B_{n}, V_{n}\right\}$ the diamond-like hierarchical lattices $[6,7]$. (See Figure 1 for the case $b=2$ ).

Bleher and Zalis [6] showed that the free energy on this lattices is discribed as follows,

$$
\mathcal{F}(T)=-\frac{J}{2}-\frac{T}{2} \sum_{n=0}^{\infty} \frac{1}{(2 b)^{n}} \log \left(1+t_{n}^{b}\right)
$$

where

$$
\left\{\begin{aligned}
t_{0} & =\exp \left(\frac{2 J}{b T}\right), \\
t_{n+1} & =f\left(t_{n}\right), \\
f(t) & =\frac{4 t^{b}}{\left(1+t^{b}\right)^{2}}
\end{aligned}\right.
$$

The map $f$ is called Migdal-Kadanoff renormalization group transformation $[4,5]$ which is a strong tool to investigate the asymptotic behavior of free energy near critical tempereture. The advantage of this model is that the renormalization group transformation can be expressed explicitly and, moreover, it turns out to be a rational map.

In [1], T. D. Lee and C. N. Yang gave an idea for the study of the singularities of free energy. Because free energy is defined as the logarithm of partition function, the singularities of free energy appear at the zeros of partition functions. But it is shown that the partition function is essentially a polynomial of $t$ with positive coefficients. So the singularities never appear on the temperature interval $[0,1]$ (remark that by change of variable, $t=\exp \left(-\frac{2 J}{b T}\right)$, temperature interval $[0, \infty]$ is mapped onto $\left.[0,1]\right)$. Lee and Yang proposed to extend the temperature $t$ to the complex plane. They thought that, letting $n \rightarrow \infty$, the zeros in the complex plane approach to some points of $[0,1]$, which would represent the phase transition point. Following this idea, we consider $\mathcal{F}$ on $\mathbf{C}$ and $f$ as a dynamical system on $\widehat{\mathbf{C}}$. 


\section{Dynamics of the Renormalization Group}

The theory of complex dynamical systems appearently developed after the works of Douady, Hubbard and Sullivan. One of the main object of this theory is to study invariant set called Julia set $J(f)$ on which the dynamics of $f$ is "chaotic". Figure 2 shows the Julia set for $b=2$.

First we consider $f$ as a dynamical system on $[0,1]$. Then,

\section{Lemma 1 (Bleher-Zalis [6])}

1. $t=0,1$ are super attractive fixed points of $f$.

2. There exists a unique repelling fixed point $t_{c}$ in $(0,1)$.

As explained in the previous section, we consider $\mathcal{F}$ as a function on $\mathbf{C}$, and $f$ as a dynamical system on $\widehat{\mathbf{C}}$. Let $\Omega_{0}$ be the immediate attractive basin of 0 . Then, we can show the following.

\section{Lemma 2 (Bleher-Lyubich [7])}

1. $\Omega_{0}$ is a Jordan domain (i.e. $\partial \Omega_{0}$ is a Jordan curve). Moreover, $\partial \Omega_{0}$ is a quasi-circle (i.e. the image of $S^{1}$ by a quasi-conformal map).

2. $\left.f\right|_{\Omega_{0}}$ is conformally conjugated to the map

$$
z \mapsto z^{b} \text { on } U=\{z \in \mathbf{C}|| z \mid<1\} .
$$

(Let us denote the conjugate map by $\varphi$.)

Since $\Omega_{0}$ is Jordan, $\varphi$ can be extended to a homeomorphism from $\bar{\Omega}_{0}$ onto $\bar{U}$. (We denote the extended map by $\varphi$ again.)

From now on, we consider

$$
F(t)=\sum_{n=0}^{\infty} \frac{1}{(2 b)^{n}} g \circ f^{n}(t), \quad g(s)=\log \left(1+s^{b}\right)
$$

instead of $\mathcal{F}$, where $f^{n}$ is the $n$-fold iterate of $f$.

Lemma 3 (Bleher-Lyubich) $F$ is analytic on $\Omega_{0}$, and $\partial \Omega_{0}$ is a natural boundary of $F$. 
From the physical point of view, we are interested in the behavior of $F^{(l)}$ (the $l$-th derivative of $F$ ) when $t$ approaches $\partial \Omega_{0}$. The $\varphi^{-1}$-image of a family of line segments $\left\{r e^{i \theta} \mid 0 \leq r \leq 1\right\}$ in $\bar{U}$ are called geodesics in $\bar{\Omega}_{0}$. Let $B_{\tau}$ denotes the geodesic ending at $\tau \in \partial \Omega_{0}$ (From the previous remark, there is bijective correspondance between end points and geodesics.) Let $\mu_{0}$ be the harmonic measure on $\partial \Omega_{0}$, i.e. $\mu_{0}=\left(\varphi^{-1}\right)_{*} \lambda$, where $\lambda$ is the normalized Lebesgue measure on $\partial U$. For $t \in B_{\tau}, l(t)$ denotes the length of $B_{\tau}$ from $t$ to $\tau$ (in the Euclidian metric). Remark that the Julia set $J(f)$ is symmetric with respect to the real axis, so $B_{t_{c}}=\left[0, t_{c}\right] \subset \mathbf{R}$.

Definition 1 For $\tau \in \partial \Omega_{0}$ and $l \in \mathbf{N}$,

$$
\alpha_{\tau}^{(l)} \equiv \lim _{\substack{t \rightarrow \tau \\ t \in B_{\tau}}} \frac{\log \left|\mathcal{F}^{(l)}(t)\right|}{-\log l(t)} .
$$

We call it the critical exponent of order $l$ along $B_{\tau}$. From the previous remark, $\alpha_{t_{c}}^{(l)}=\alpha^{(l)}$ which was defined in the introduction.

In [7], Bleher and Lyubich proved the following.

Theorem (Bleher-Lyubich) Let $b>2$. Then,

1. $F^{(2)}(t)$ is not continuous up to $\partial \Omega_{0}$.

2. For $\mu_{0}$-almost all $\tau \in \partial \Omega_{0}$

$$
\alpha_{\tau}^{(2)}=1-\frac{\log 2}{\log b} .
$$

Here one question arises. Is this result valid for $t_{c} \in \partial \Omega_{0}$ ? Because, physically, real critical exponent $\alpha_{t_{c}}^{(l)}$ is the most important. Theorem B tells us the answer of this question.

\section{Properties of the Maximal Entropy Mea- sure}

Here we introduce a "natural" measure $\mu$ on $J(f)$ in order to analyse the properties of the free energy near $\partial \Omega_{0}$. This measure was, for the first time, introduced by Brolin [10] for the polynomial case. 
First, it is not difficult to see that all the critical points of $f$ are eventually mapped to the superstable fixed points 0 or 1 . So, the dynamics of $f$ on $J(f)$ is expanding (i.e. there exists constants $C>0$ and $\lambda>1$ such that $\left\|d f^{n}(z)\right\| \geq C \cdot \lambda^{n}$ for all $n \geq 0$, and all $\left.z \in J(f)\right)$.

Thus, from Bowen-Ruelle-Sinai's theory there exists a unique equilibrium state $\mu$ satisfies the variational principle for potential $\rho \equiv 0$,

$$
P_{f}(0)=h_{\mu}(f)=\sup _{\nu \in M(f)} h_{\nu}(f)
$$

where $M(f)$ denotes all $f$-invariant probability measure on $J(f) . P_{f}(0)$ is just the topological entropy, so $\mu$ is called the maximal entropy measure. This measure has many properties. Let $\operatorname{Exc}(f)$ be the exceptional points of $f$. Remark that $\operatorname{deg} f=2 b$.

\section{Proposition 1 (Freire-Lopes-Mañé [11], Mañé [12], Lyubich [13])}

1. $h_{\mu}(f)=\log 2 b$.

2. $\operatorname{supp}(\mu)=J(f)$.

3. For all $a \in \widehat{\mathbf{C}} \backslash \operatorname{Exc}(f)$, let

$$
\mu_{n} \equiv \frac{1}{(2 b)^{n}} \sum_{i=1}^{(2 b)^{n}} \delta_{z_{i}^{(n)}(a)}
$$

where $\delta_{x}$ is the Dirac measure at $x$ and $\left\{z_{i}^{(n)}(a)\right\}_{i=1}^{2 b}$ are the solutions of $f^{n}(z)=$ a counting multiplicity. Then

$$
\mu_{n} \rightarrow \mu \quad \text { weakly as } n \rightarrow \infty \text {. }
$$

4.

$$
\mu(f(A))=(2 b) \cdot \mu(A)
$$

for any Borel set $A$ where $\left.f\right|_{A}$ is injective. Conversely, the maximal entropy measure is the unique $f$-invariant probability measure satisfies the above equation. 


\section{Proofs of the Main Theorems}

In this section we sketch the proofs of our theorems.

\section{Proof of Theorem A.}

Consider the functional equation

$$
E(t)=\frac{1}{2 b} E \circ f(t)+g(t)
$$

It is easy to see that both expressions satisfy the above equation. So we must claim the uniqueness of the continuous solution $E(t)$ of the above equation satisfying $E(0)=0$. Let $G(t)$ be the difference of the two expressions. Then $G$ must satisfy

$$
\frac{1}{2 b} G \circ f(t)-G(t)=0, \quad G(0)=0 .
$$

Assume $G\left(t_{0}\right) \neq 0$ for some $t_{0} \in \Omega_{0}$. Then, Using this equation inductively, we get

$$
G \circ f^{n}\left(t_{0}\right)=(2 b)^{n} \cdot G\left(t_{0}\right) .
$$

Because $t_{0} \in \Omega_{0}, f^{n}\left(t_{0}\right)$ goes to 0 as $\mathrm{n}$ increases. Thus by the continuity of $G, G \circ f^{n}\left(t_{0}\right) \rightarrow 0$. But if we assume $G\left(t_{0}\right) \neq 0$ for some $t_{0} \in \Omega_{0}$, then $(2 b)^{n} \cdot G\left(t_{0}\right)$ goes to infinity as $n \rightarrow \infty$. This is a contradiction.

Remark 1 The functional equation often generates continuous but nowhere differentiable functions including Takagi's function and Weierstrass's function. For details, see [14].

Next we establish the relationship between real critical exponent and the maximal entropy measure, using the representation in Theorem A. Remark that $f^{\prime}\left(t_{c}\right)>1$.

\section{Proof of Theorem B.}

First remark that, in the integral representation, the integral near $t_{c}$ dominates $F^{(l)}(t)$. Let $r>0$ be small enough so that we can linearize $f(t)$ on $B_{r}\left(t_{c}\right)=\left\{z \in \mathbf{C}|| z-t_{c} \mid \leq r\right\}$ and fix it. Let $J_{r} \equiv J(f) \cap B_{r}\left(t_{c}\right)$, and take arbitrary $p_{0} \in(0, t) \cap B_{r}\left(t_{c}\right)$. Define a sequence $p_{n} \in\left(p_{n-1}, t_{c}\right)$ so that 
$p_{n-1}=f\left(p_{n}\right)$. Let $f^{-1}$ be the inverse branch on $J_{r}$ which fixes $t_{c}$. Consider the ratio,

$$
\frac{F^{(l)}\left(p_{n+1}\right)}{F^{(l)}\left(p_{n}\right)}=\frac{c_{l} \int_{f^{-1}\left(J_{r}\right)} \frac{d \mu(t)}{\left(t-p_{n+1}\right)^{l}}+c_{l} \int_{J(f) \backslash f^{-1}\left(J_{r}\right)} \frac{d \mu(t)}{\left(t-p_{n+1}\right)^{l}}}{c_{l} \int_{J_{r}} \frac{d \mu(t)}{\left(t-p_{n}\right)^{l}}+c_{l} \int_{J(f) \backslash J_{r}} \frac{d \mu(t)}{\left(t-p_{n}\right)^{l}}}
$$

Let $t^{\prime}=f(t)$. Then,

$$
\int_{f^{-1}\left(J_{r}\right)} \frac{d \mu(t)}{\left(t-p_{n+1}\right)^{l}}=\frac{1}{2 b} \int_{J_{r}} \frac{d \mu\left(t^{\prime}\right)}{\left(f^{-1}\left(t^{\prime}\right)-f^{-1}\left(p_{n}\right)\right)^{l}}
$$

From the definition of the derivative

$$
\frac{1}{f^{-1}\left(t^{\prime}\right)-f^{-1}\left(p_{n}\right)} \approx \frac{f^{\prime}\left(t_{c}\right)}{t^{\prime}-p_{n}}
$$

So the ratio $(5.3)$ goes to

$$
\frac{\left(f^{\prime}\left(t_{c}\right)\right)^{l}}{2 b}
$$

as $n$ tends to infinity. Taking logarithms, we get

$$
\log F^{(l)}\left(p_{n+1}\right) \approx n \log \frac{\left(f^{\prime}\left(t_{c}\right)\right)^{l}}{2 b}+\log F^{(l)}\left(p_{0}\right)
$$

On the other hand, we have

$$
\log \left(t_{c}-p_{n+1}\right) \approx \log \left(t_{c}-p_{0}\right)-n \log f^{\prime}\left(t_{c}\right) .
$$

From the equations (5.5) and (5.6), it is not difficult to deduce our statement from this.

Remark 2 A similar equation has been already conjectured in [9]. Indeed, we need not use the integral representation to calculate the critical exponent itself. 


\section{Local Similarity of $\mu$}

What does $\log 2 b / \log f^{\prime}\left(t_{c}\right)$ in Theorem B mean? First, consider, for example, the Sierpinski gasket. When we enlarge the size of the Sierpinski gasket twice, the "area" (rigorously speaking, the Hausdorff measure) becomes three times. Thus, the similarity dimension of the Sierpinski gasket equals to $\log 3 / \log 2$. This is the fundamental idea of similarity dimension.

In our case, if we linearize $f$ near $t_{c}$, then the equation (4.1) becomes

$$
\mu(L(V)) \approx(2 b) \cdot \mu(V)
$$

where $L(t) \equiv f^{\prime}\left(t_{c}\right) \cdot\left(t-t_{c}\right)+t_{c}$ is the linearization of $f$, and $V$ is a neighborhood of $t_{c}$. See Figure 3 where we can see the fractal structure of $J(f)$ near $t_{c}$, and convince that the above equation is quite precise. This situation is just the same as the case of the Sierpiński gasket. That is, when we enlarge the size of $V f^{\prime}\left(t_{c}\right)$-times, then the measure $\mu(V)$ becomes about $2 b$ times. So $\log 2 b / \log f^{\prime}\left(t_{c}\right)$ is supposed to represent a similarity of $\mu$ near $t_{c}$. Thus, Theorem B states that the critical exponent reflects the local similarity of the maximal entropy measure.

Remark 3 The following remark is due to Dr. Kokubu on a connection between the results of Bleher-Lyubich and the author.

If we rewrite the result of Bleher-Lyubich in our fashion, we get

$$
\alpha_{\tau}^{(2)}=2-\frac{\log 2 b}{\log b} \text {. }
$$

So in both cases the critical exponent shows the following form

$$
l-\frac{\text { topological entropy of } f}{\text { Lyapunov exponent at } \tau} \text {. }
$$

Applying something like Pesin's formula [15], we get

$$
\alpha_{\tau}^{(l)}=l \text {-local dimension of the maximal entropy measure. }
$$

Acknowledgement. The author express his gratitude to Prof. M. Yamaguti and Prof. M. Hata for their encouragement and advices. He also thanks to Dr. Kokubu and Dr. Takahashi for many useful comments and fruitful discussions. 


\section{References}

[1] Lee, T. D., Yang, C. N. Statistical theory of equations of state and phase transitions, II. Lattice gas and Ising model. Phys. Rev. 87, 410-419 (1952).

[2] Wilson, K. G., Kogut, J. The renormalization group and the $\epsilon$-expansion. Phys. Repts. 12C, 75 (1974).

[3] Fisher, M. E. The renormalization group in the theory of critical behavior. Rev. Mod. Phys. 46, 597 (1974).

[4] Migdal, A. A. Reccurence equation in gauge field theory. JETP 69, 810$822,1457-1467$ (1975).

[5] Kadanoff, L. P. Notes on Migdal's recursion formurae. Ann. Phys. 100, 359-394 (1976).

[6] Bleher, P. M., Zalis, E. Asymptotics of the susceptibility for the Ising model on the hierarchical lattices. Commun. Math. Phys. 120, 409-436 (1989).

[7] Bleher, P. M., Lyubich, M. Yu. Julia sets and complex singulalities in hierarchical Ising models. Commun. Math. Phys. 141, 453-474 (1991).

[8] Derrida, B., De Seze, L., Itzykson, C. Fractal structure of zeros in hierarchical lattices. J. Stat. Phys. 30, 559-570 (1983).

[9] Derrida, B., Eckmann, J.-P., Erzan, A. Renormalization groups with periodic and aperiodic orbits. J. Phys. A: Math. Gen. 16, 893-906 (1983).

[10] Brolin, H. Invariant sets under iteration of rational functions. Ark. Mat. 6, 103-144 (1965).

[11] Freire, A., Lopes, A., Mañé, R. An invariant measure for rational maps. Bol. Soc. Bras. Mat. 14, 45-62 (1983).

[12] Mañé, R. On the uniqueness of the maxmizing measure for rational maps. Bol. Soc. Bras. Mat. 14, 27-43 (1983). 
[13] Lyubich, M., Entropy properties of rational endomorphisms of the Riemann sphere. Erg. Th. Dyn. Syst. 3, 351-385 (1983).

[14] Yamaguti, M., Hata, M. Weierstrass's function and chaos. Hokkaido Math. J. 12, 333-342 (1983).

[15] Pesin, Y. Characteristic Lyapunov exponents and smooth ergodic theory. Russian Math. Surveys 32, 55-114 (1977).

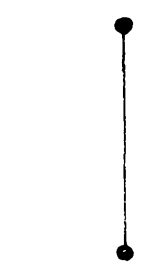

$I_{0}=\left\{B_{0}, V_{0}\right\}$

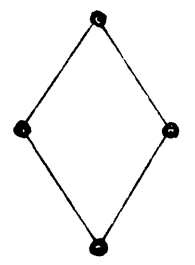

$$
\vec{L}_{1}=\left\{B_{1}, V_{1}\right\}
$$

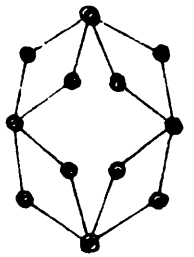

$\vec{I}_{2}=\left\{B_{2}, V_{2}\right\}$ 


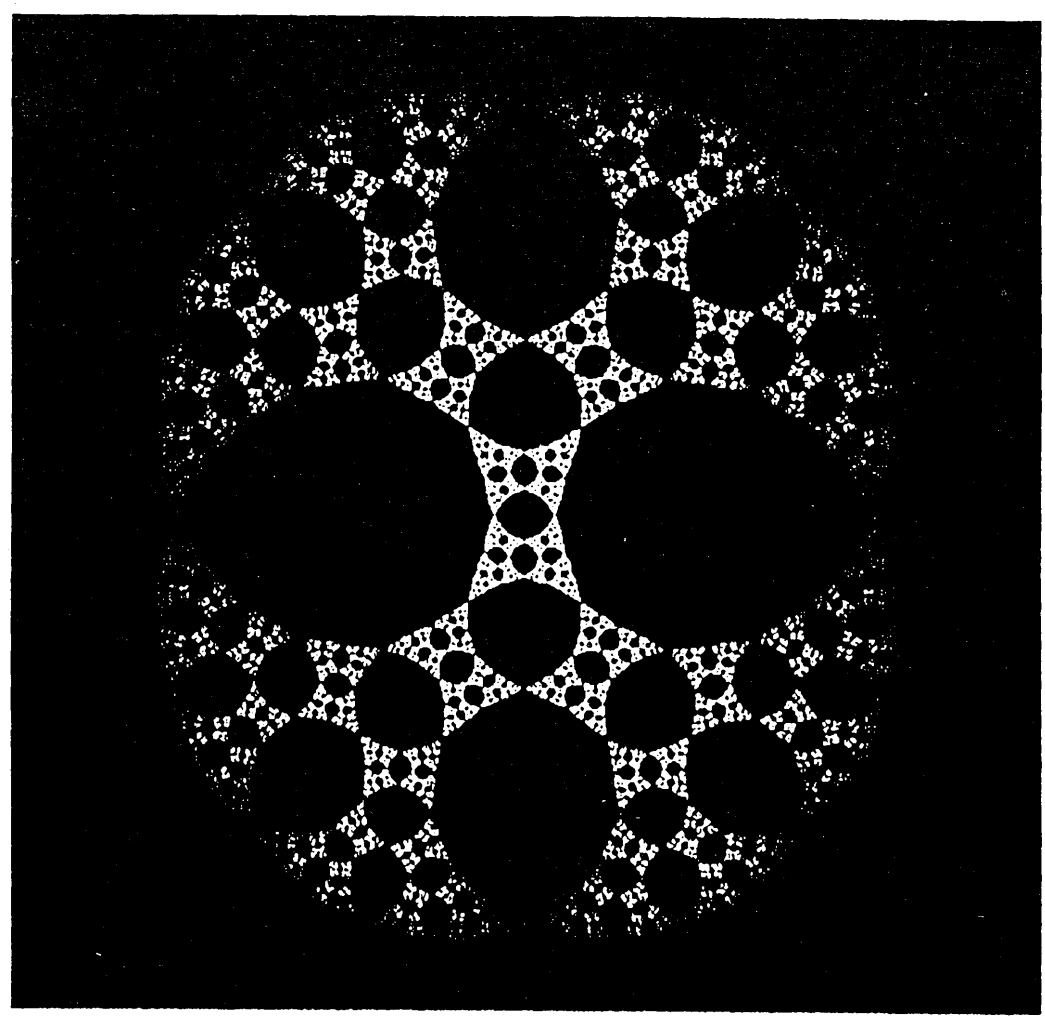

Figure 2
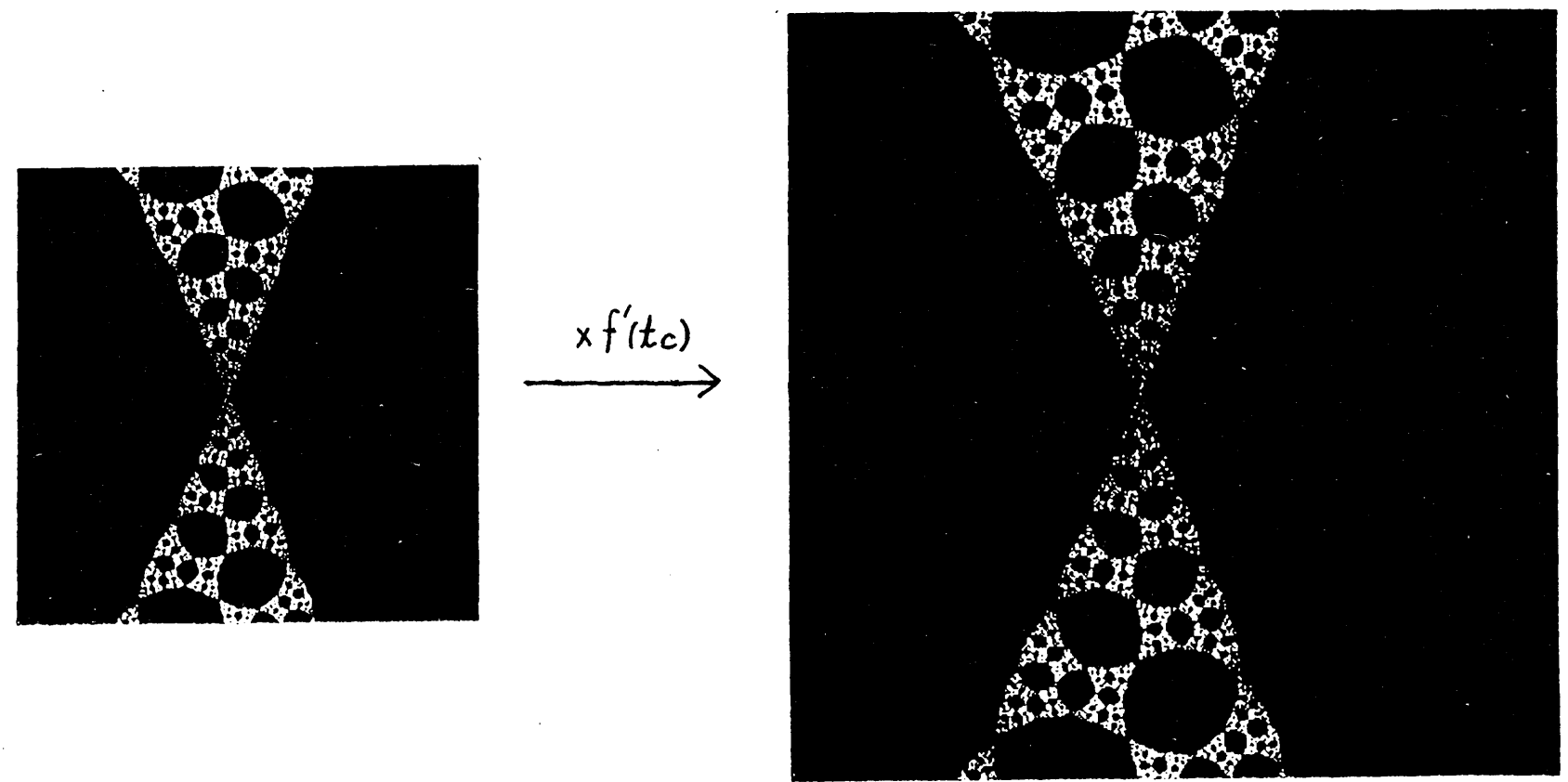

Figure 3

$\dot{x}$. These figures are written by Mr. Yamagishi (Ryukoku). The author thanks to him. 